\title{
Combinatorics of symplectic invariant tensors
}

\author{
Martin Rubey 1 tand Bruce W. Westbury $\|^{\#}$ \\ ${ }^{1}$ Fakultät für Mathematik und Geoinformation, TU Wien, Austria \\ ${ }^{2}$ Department of Mathematics, University of Warwick, Coventry, CV4 7AL
}

\begin{abstract}
An important problem from invariant theory is to describe the subspace of a tensor power of a representation invariant under the action of the group. According to Weyl's classic, the first main (later: 'fundamental') theorem of invariant theory states that all invariants are expressible in terms of a finite number among them, whereas a second main theorem determines the relations between those basic invariants.

Here we present a transparent, combinatorial proof of a second fundamental theorem for the defining representation of the symplectic group $\operatorname{Sp}(2 n)$. Our formulation is completely explicit and provides a very precise link to $(n+1)$ noncrossing perfect matchings, going beyond a dimension count. As a corollary, we obtain an instance of the cyclic sieving phenomenon.

Résumé. Une probléme importante de la théorie des invariantes est de décrire le sous espace d'une puissance tensorielle d'une répresentation invariant à l'action de la groupe. Suivant la classique de Weyl, la théoreme fondamentale premiere pour la répresentation standard de la group sympléctique dit que toutes invariantes peuvent être expriment entre un nombre fini d'entre eux. Ainsi, une théoreme fondamentale seconde determine les rélations entre ces invariantes basiques.

Ici, nous présentons une preuve transparente d'une théoreme fondamentale seconde pour la répresentation standard de la groupe sympléctique $\operatorname{Sp}(2 n)$. Notre formulation est completement explicite est elle provide un lien tres précis avec les couplages parfaites $(n+1)$-noncroissants, plus précis qu'un denombrement de la dimension. Comme corollaire nous exhibons une phénomène du crible cyclique.
\end{abstract}

Keywords: invariant tensors, cyclic sieving phenomenon, matchings, classical groups

Dedicated to Mia and George

\section{Introduction}

The primary motivation of this article is a specific example of the cyclic sieving phenomenon, as introduced by Reiner, Stanton and White [11], concerning the set $X(r, n)$ of $(n+1)$-noncrossing perfect matchings of $\{1, \ldots, 2 r\}$. These are perfect matchings that do not contain any set of $(n+1)$ pairs $\left(a_{1}, b_{1}\right), \ldots,\left(a_{n+1}, b_{n+1}\right)$ with $a_{1}<\cdots<a_{n+1}<b_{1}<\cdots<b_{n+1}$.

\footnotetext{
†Email: Martin.Rubey@tuwien.ac.at

‡Email: Bruce.Westbury@warwick.ac.uk 
A standard visualisation of a perfect matching is obtained by placing the numbers from 1 to $2 r$ in this order on a circle and connecting two numbers by a straight edge if they form a pair in the matching. Using this visualisation, a perfect matching is $(n+1)$-noncrossing if at most $n$ edges cross mutually. The set $X(r, n)$ carries a natural action of the cyclic group of order $2 r$, given by $\rho: i \mapsto i(\bmod 2 r)+1$, i.e., the rotation map.

With this definition we can state what might be taken as the main theorem of this article:

Theorem. Let

$$
P(q)=\sum_{T} q^{\operatorname{maj} T}
$$

where the sum is over all $n$-symplectic oscillating tableaux of length $2 r$ and weight 0 and maj $T$ is the sum of the positions of the descents in $T$, as defined in [13].

Then the triple $(X(r, n), \rho, P(q))$ exhibits the cyclic sieving phenomenon.

However, we would like to stress that this is merely a by-product of the approach taken in this article to the invariant theory of the classical groups, aiming at tying together their representation theory and combinatorics in a concrete fashion.

In our main example we consider tensor powers of the defining representation $V$ of the symplectic group $\operatorname{Sp}(2 n)$, the group acting diagonally. The symmetric group $\mathfrak{S}_{r}$ also acts on $\otimes^{r} V$, by permuting tensor positions. This action is inherited by the subspace of $\otimes^{r} V$ which is invariant under the action of $\operatorname{Sp}(2 n)$.

An effective method to understand both the tensor powers $\otimes^{r} V$ and their invariant subspaces is to use diagram categories. In the case at hand this is a specialisation $\mathrm{D}_{\mathrm{Sp}(2 n)}$ of the Brauer category. The objects of this category are the natural numbers and $\operatorname{Hom}_{\mathrm{D}_{\mathrm{Sp}(2 n)}}(r, s)$ is the vector space whose basis is the set of perfect matchings of $\{1, \ldots, r+s\}$, with a natural composition, see Section 2

The connection to the tensor powers of the defining representation of $\operatorname{Sp}(2 n)$ is established by considering the category $\mathrm{T}_{\mathrm{Sp}(2 n)}$ of invariant tensors of the defining representation of the symplectic group, and a certain functor $\mathrm{ev}_{\mathrm{Sp}(2 n)}$ from the diagram category $\mathrm{D}_{\mathrm{Sp}(2 n)}$ to $\mathrm{T}_{\mathrm{Sp}(2 n)}$, see Definition 2.1.

The first fundamental theorem, Weyl [21. Theorem (6.1A)], due to Brauer [1], is equivalent to the statement that this functor is full, that is, surjective on morphisms. What is traditionally named second fundamental theorem is a description of the kernel of the linear map from $\operatorname{Hom}_{\mathrm{D}_{\mathrm{Sp}(2 n)}}(r, s)$ to $\operatorname{Hom}_{\mathrm{T}_{\mathrm{Sp}(2 n)}}(r, s)$ induced by $\mathrm{ev}_{\mathrm{Sp}(2 n)}$. We provide the following explicit formulation, which is also a key ingredient for the proof of Theorem 3.5

Theorem. Let $\mathrm{Pf} \in \mathrm{Hom}_{\mathrm{D}_{\mathrm{Sp}(2 n)}}(0,2(n+1))$ be the sum of all perfect matchings of $\{1, \ldots, 2(n+1)\}$ and let $\mathrm{Pf}^{(n)}$ be the 'pivotal symmetric $\dagger^{\dagger}$ ideal generated by $\mathrm{Pf}$. Then the categories $\mathrm{D}_{\mathrm{Sp}(2 n)} / \mathrm{Pf}^{(n)}$ and $\mathrm{T}_{\mathrm{Sp}(2 n)}$ are isomorphic. Moreover, the set $X(r, n)$ is a basis of $\operatorname{Hom}_{\mathrm{D}_{\mathrm{Sp}(2 n)} / \mathrm{Pf}^{(n)}}(0,2 r)$.

Remarkably, a well-known result due to Sundaram [16] states that the cardinality of $X(r, n)$ equals the dimension of $\operatorname{Hom}_{\mathrm{Sp}(2 n)}\left(\mathbb{C}, \otimes^{2 r} V\right)$. In fact, Sundaram also computed the Frobenius character of this representation, and, more generally, of $\operatorname{Hom}_{\mathrm{Sp}(2 n)}\left(W(\mu), \otimes^{r} V\right)$ for any irreducible representation $W(\mu)$ of the symplectic group $\mathrm{Sp}(2 n)$.

We note that the same categorical setup was employed by Lehrer and Zhang [7] to show that $\operatorname{Pf}^{(n)}$ restricted to the Brauer algebra $\operatorname{Hom}_{\mathrm{D}_{\mathrm{Sp}(2 n)}}(r, r)$ is in fact generated as a two-sided ideal by a single element.

$\dagger$ We define pivotal and symmetric ideals in [14], and restrict ourselves here to down-to-earth language in Definitions 2.10 and 2.11 


\section{Fundamental theorems}

The theorems of this section connect the Brauer category with the invariant theory of the defining representation of the symplectic group by providing an explicit isomorphism of categories.

First we recall the definition of the Brauer category. Let $D(r, s)$ be the set of perfect matchings on $[r] \amalg[s]$. In particular, $D(r, s)=\emptyset$ if $r+s$ is odd. An element of $D(r, s)$ is visualised as a set of $(r+s) / 2$ strands drawn in a rectangle, with $r$ endpoints on the top edge of the rectangle and $s$ endpoints on the bottom edge.

The composition of two diagrams $x \in D(r, s)$ and $y \in D(s, t)$ is obtained by identifying the points on the bottom edge of $x$ with the points on the top edge of $y$. Let $c(x, y)$ be the number of closed loops in the resulting diagram and let $x \circ y \in D(r, t)$ be the perfect matching obtained by removing all closed loops.

The Brauer category $\mathrm{D}_{\mathrm{Br}}$ has objects $\mathbb{N}$. Its morphisms $\operatorname{Hom}_{\mathrm{D}_{\mathrm{Br}}}(r, s)$ are given by the free $\mathbb{Z}[\delta]$-module with basis $D(r, s)$. The composition of morphisms is defined on basis elements by

$$
x \cdot y=\delta^{c(x, y)} x \circ y
$$

and extended bilinearly. The endomorphism algebra $\operatorname{Hom}_{\mathrm{D}_{\mathrm{Br}}}(r, r)$ is the Brauer algebra, which will be denoted by $D_{r}$ from now on. Finally, let $\mathrm{D}_{\mathrm{Sp}(2 n)}$ be the specialisation of $\mathrm{D}_{\mathrm{Br}}$ obtained by applying the homomorphism $\mathbb{Z}[\delta] \rightarrow \mathbb{C}, \delta \mapsto-2 n$.

The Brauer category also has a tensor product. The tensor product $x \otimes y$ of two diagrams $x$ and $y$ is obtained by putting the diagrams side-by-side, identifying the right hand edge of $x$ with the left hand edge of $y$.

We now turn to the definition of the category of invariant tensors $\mathrm{T}_{\mathrm{Sp}(2 n)}$. Recall that the symplectic group $\operatorname{Sp}(V)$ is the group of linear transformations of a $2 n$-dimensional complex vector space $V$ that preserve a non-degenerate skew-symmetric bilinear form $\langle$,$\rangle . However, the categorical machinery we$ use requires that a symmetric bilinear form is preserved. Therefore, we regard $V$ as an odd vector space, which has precisely the desired effect. Among the further consequences we remark that in this convention the dimension of $V$ is $-2 n$ and symmetric and exterior powers are interchanged.

Let $\left\{b_{1}, \ldots, b_{2 n}\right\}$ be a basis of $V$ and let $\left\{\bar{b}_{1}, \ldots, \bar{b}_{2 n}\right\}$ be the dual basis, so $\left\langle\bar{b}_{i}, b_{j}\right\rangle=\delta_{i, j}$. Let $\mathrm{T}_{\mathrm{Sp}(2 n)}$ be the category whose objects are again the natural numbers and whose morphisms $\operatorname{Hom}_{\mathrm{T}_{\mathrm{Sp}(2 n)}}(r, s)$ are the equivariant maps

$$
\operatorname{Hom}_{\mathrm{Sp}(2 n)}\left(\otimes^{r} V, \otimes^{s} V\right)=\left\{\phi: \otimes^{r} V \rightarrow \otimes^{s} V \mid g \cdot \phi(\mathbf{v})=\phi(g \cdot \mathbf{v}), g \in \operatorname{Sp}(2 n)\right\} .
$$

Note that the invariant subspace of $\otimes^{r} V$ is precisely $\operatorname{Hom}_{\mathrm{T}_{\mathrm{Sp}(2 n)}}(0, r)=\operatorname{Hom}_{\mathrm{Sp}(2 n)}\left(\mathbb{C}, \otimes^{r} V\right)$.

The connection between $\mathrm{D}_{\mathrm{Sp}(2 n)}$ and $\mathrm{T}_{\mathrm{Sp}(2 n)}$ is established by a functor we now define:

Definition 2.1. Let $\operatorname{ev}_{\mathrm{Sp}(2 n)}$ be the evaluation functor $\mathrm{D}_{\mathrm{Sp}(2 n)} \rightarrow \mathrm{T}_{\mathrm{Sp}(2 n)}$. Explicitly, $\mathrm{ev}_{\mathrm{Sp}(2 n)}$ sends the object $r \in \mathbb{N}$ to $\otimes^{r} V$. It is defined on the generators by

$$
\begin{aligned}
& \operatorname{ev}_{\mathrm{Sp}(2 n)}(\searrow)=u \otimes v \mapsto-v \otimes u \\
& \operatorname{ev}_{\operatorname{Sp}(2 n)}(\cap)=1 \mapsto \sum_{i} b_{i} \otimes \bar{b}_{i} \\
& \operatorname{ev}_{\mathrm{Sp}(2 n)}(\bigcup)=u \otimes v \mapsto\langle u, v\rangle .
\end{aligned}
$$

and is then uniquely determined by the following three properties 
- it is a functor, so $\operatorname{ev}_{\mathrm{Sp}(2 n)}(x \cdot y)=\operatorname{ev}_{\mathrm{Sp}(2 n)}(x) \circ \operatorname{ev}_{\mathrm{Sp}(2 n)}(y)$,

- it respects tensor products, so $\operatorname{ev}_{\mathrm{Sp}(2 n)}(x \otimes y)=\operatorname{ev}_{\mathrm{Sp}(2 n)}(x) \otimes \operatorname{ev}_{\mathrm{Sp}(2 n)}(y)$,

- it is linear.

The first fundamental theorem for the symplectic group can now be stated as follows:

Theorem 2.2. [1], [21, Theorem (6.1A)] For all $n>0$ the functor $\operatorname{ev}_{\mathrm{Sp}(2 n)}: \mathrm{D}_{\mathrm{Sp}(2 n)} \rightarrow \mathrm{T}_{\mathrm{Sp}(2 n)}$ is full.

In the remainder of this section we provide an explicit description of the kernel of $\operatorname{ev}_{\mathrm{Sp}(2 n)}$ as an ideal in the Brauer category. We will denote this ideal with $\mathrm{Pf}^{(n)}$ because of its intimate connection to the Pfaffian. Moreover we obtain a simple basis for the vector space $\operatorname{Hom}_{\mathrm{D}_{\mathrm{Sp}(2 n)} / \mathrm{Pf}(n)}(0, r)$. This basis is preserved by rotation, which we will use in Section 3 to exhibit a cyclic sieving phenomenon.

The fundamental object involved is an idempotent $E(n+1)$ of the Brauer algebra $D_{n+1}$ for which we have $\operatorname{ev}_{\mathrm{Sp}(2 n)}(E(n+1))=0$. This element can be characterised as follows.

Let $\rho$ be the one dimensional representation of $D_{n+1}$ which on diagrams is given by

$$
\rho(x)= \begin{cases}1 & \text { if } \operatorname{pr}(x)=n+1 \\ 0 & \text { if } \operatorname{pr}(x)<n+1\end{cases}
$$

where $\operatorname{pr}(x)$ is the propagating number of a diagram $x$, i.e., the number of strands connecting a point on the top edge with a point on the bottom edge.

The element $E(n+1)$ of $D_{n+1}$ is determined, up to scalar multiple, by the properties

$$
x E(n+1)=\rho(x) E(n+1)=E(n+1) x .
$$

It follows that $E(n+1)$ can be scaled so that it is idempotent and these properties now determine $E(n+1)$. It is clear that $E(n+1)$ is a central idempotent and that its rank equals one.

We will give two constructions of $E(n+1)$. The first is as a simple linear combination of diagrams.

\section{Definition 2.3.}

$$
E(n+1)=\frac{1}{(n+1) !} \sum_{x \in D(n+1, n+1)} x
$$

Lemma 2.4. For $\delta=-2 n$, the element $E(n+1)$ in Definition 2.3 satisfies the properties (2).

Proof: If $\operatorname{pr}(x)=n+1$ then $x$ acts as a permutation on the set of diagrams $D(n+1, n+1)$, so this case is clear.

Let $\mathrm{J}_{r}(p)$ be the ideal of the Brauer algebra $D_{r}$ generated by the diagrams with propagating number at most $p$. The elements $u_{i}, 1 \leq i \leq n$ generate the ideal $\mathrm{J}_{n+1}(n)$ so it is sufficient to show that $u_{i} E(n+1)=0$ and $E(n+1) u_{i}=0$ for $1 \leq i \leq n$. We will now show that $u_{i} E(n+1)=0$. The case $E(n+1) u_{i}=0$ is similar.

It is clear that $u_{i} E(n+1)$ is a linear combination of diagrams which contain the pair $(i, i+1)$. Hence it is sufficient to show that the coefficient of each of these diagrams is 0 . Let $x$ be a diagram which contains the pair $(i, i+1)$. The set $\left\{y \in D(n+1, n+1): u_{i} y=x\right\}$ has cardinality $2 n$ and there is precisely one diagram $z$ with $u_{i} z=\delta x$. Therefore the coefficient of $x$ is $\delta+2 n$, which vanishes for $\delta=-2 n$. 
The set of $2 n$ diagrams is constructed as follows. The diagram $x$ contains $n$ pairs other than $(i, i+1)$. For each of these pairs we construct two elements of the set. Take the pair $(u, v)$, and replace the two pairs $(i, i+1)$ and $(u, v)$ by $(i, u)$ and $(i+1, v)$ and by $(i+1, u)$ and $(i, v)$ keeping all the remaining pairs.

However it is not clear from this construction that $\operatorname{ev}_{\mathrm{Sp}(2 n)}(E(n+1))=0$. We now give an alternative construction which does make this clear.

Definition 2.5. For $1 \leq i \leq n$ define $u_{i} \in D(n+1, n+1)$ to consist of the pairs $\left(a, a^{\prime}\right)$ for $a \notin\{i, i+1\}$ together with the pairs $(i, i+1)$ and $\left(i^{\prime},(i+1)^{\prime}\right)$ and define $s_{i} \in D(n+1, n+1)$ to consist of the pairs $\left(a, a^{\prime}\right)$ for $a \notin\{i, i+1\}$ together with the pairs $\left(i,(i+1)^{\prime}\right)$ and $\left(i^{\prime}, i+1\right)$ :
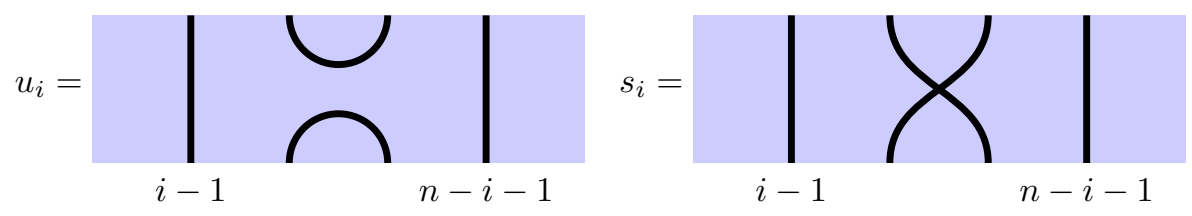

Definition 2.6. For $k \in \mathbb{Z}$ and $1 \leq i \leq n$ define $R_{i}(k) \in D_{n+1}$ by

$$
R_{i}(k)=\frac{1}{k+1}\left(1+k s_{i}-\frac{2 k}{\delta+2 k-2} u_{i}\right)
$$

Proposition 2.7. These elements satisfy

$$
\begin{aligned}
R_{i}(h) R_{i+1}(h+k) R_{i}(k) & =R_{i+1}(k) R_{i}(h+k) R_{i+1}(h) \\
R_{i}(h) R_{j}(k) & =R_{j}(k) R_{i}(h) \quad \text { for }|i-j|>1
\end{aligned}
$$

Proof: The second relation is clear. The first relation is known as the Yang-Baxter equation and is checked by a direct calculation. This calculation can be carried out using generators and relations or by using a faithful representation.

Definition 2.8. The element $E(n+1)$ is defined recursively by

$$
E(n+1)=E(n) R_{n}(n) E(n)
$$

There are other equivalent definitions, for example,

$$
\begin{aligned}
& E(n+1)=E(n) R_{n}(n) R_{n-1}(n-1) \ldots R_{1}(1) \\
& E(n+1)=R_{1}(1) R_{2}(2) \ldots R_{n}(n) E(n)
\end{aligned}
$$

The proof that these definitions are equivalent and the proof that for $\delta=-2 n$ this element satisfies the properties (2) are both calculations using the Yang-Baxter equation Proposition 2.7.

Proposition 2.9.

$$
\operatorname{ev}_{\operatorname{Sp}(2 n)}(E(n+1))=0
$$


Proof: Because $\mathrm{ev}_{\mathrm{Sp}(2 n)}$ is a functor, $\operatorname{ev}_{\mathrm{Sp}(2 n)}(E(n+1))$ is an idempotent in $\mathrm{T}_{\mathrm{Sp}(2 n)}$, too. Moreover, $\mathrm{ev}_{\mathrm{Sp}(2 n)}$ preserves the trace of morphisms. Recall that the (diagrammatic) trace $\operatorname{tr}_{n}$ of a diagram $\alpha$ in $D_{n}$ is defined as

$$
\eta_{2 n} \cdot\left(\alpha \otimes \mathrm{id}_{n}\right) \cdot \eta_{2 n}^{*}
$$

where $\eta_{2 n} \in D(0,2 n)$ is the diagram that consists of $n$ nested arcs. The following properties are easily verified.

$$
\begin{aligned}
\operatorname{tr}_{n+1}\left(\alpha \otimes \mathrm{id}_{1}\right) & =\delta \operatorname{tr}_{n} \alpha \\
\operatorname{tr}_{n+1} \alpha s_{n} \beta & =\operatorname{tr}_{n} \alpha \beta \\
\operatorname{tr}_{n+1} \alpha u_{n} \beta & =\operatorname{tr}_{n} \alpha \beta
\end{aligned}
$$

for $\alpha, \beta$ diagrams on $n$ strings.

The rank of an idempotent is equal to its trace so it is sufficient to show that $\operatorname{tr}_{n+1} E(n+1)=0$ for $\delta=-2 n$.

We now compute the trace by expanding $R_{n}(n)=\frac{1}{n+1}\left(1+n s_{n}+n u_{n}\right)$ :

$$
\operatorname{tr}_{n+1} E(n+1)=\frac{1}{n+1}(\delta+n+n) \mathbf{t r}_{n+1} E(n) .
$$

Substituting $-2 n$ for $\delta$ gives $\operatorname{tr}_{n+1} E(n+1)=0$.

We will now describe the kernel of $\operatorname{ev}_{\mathrm{Sp}(2 n)}$ in terms of diagrammatic Pfaffians.

Definition 2.10 ([3, Definition 3.4 (b)]). Let $\mathcal{S}$ be a $2(n+1)$-element subset of $[r] \amalg[s]$ and let $f$ be a perfect matching of $[r] \amalg[s] \backslash \mathcal{S}$. Then the diagrammatic Pfaffian of order $2(n+1)$ corresponding to $f$ is

$$
\operatorname{Pf}(f)=\sum_{s \text { a perfect matching of } \mathcal{S}}(s \cup f) .
$$

Note that for $r, s=n+1$ and $f=\emptyset$ we have, by Definition 2.3. $E(n+1)=\frac{1}{(n+1) !} \operatorname{Pf}(\emptyset)$.

Definition 2.11. For $r, s \geq 0$, the subspace $\operatorname{Pf}^{(n)}(r, s)$ in the ideal $\mathrm{Pf}^{(n)}$ of $\mathrm{D}_{\mathrm{Sp}(2 n)}$ is spanned by the set $\operatorname{Pf}^{(n)}(r, s)=\{\operatorname{Pf}(f): f$ a perfect matching of a subset of $[r] \amalg[s]$ of cardinality $r+s-2(n+1)\}$.

Definition 2.12. Let $\overline{\operatorname{ev}}_{\mathrm{Sp}(2 n)}: \mathrm{D}_{\mathrm{Sp}(2 n)} / \mathrm{Pf}^{(n)} \rightarrow \mathrm{T}_{\mathrm{Sp}(2 n)}$ be the functor that factors ev $\mathrm{vep}_{\mathrm{Sp}(2 n)}$ through the quotient.

We can now state the main theorem of this section, also known as the second fundamental theorem for the symplectic group:

Theorem 2.13. The functor $\overline{\mathrm{ev}}_{\mathrm{Sp}(2 n)}: \mathrm{D}_{\mathrm{Sp}(2 n)} / \mathrm{Pf}^{(n)} \rightarrow \mathrm{T}_{\mathrm{Sp}(2 n)}$ is an isomorphism of categories.

Proof: Since $\overline{\mathrm{ev}}_{\mathrm{Sp}(2 n)}$ is obviously bijective on objects and full by the first fundamental theorem, it is sufficient to show

$$
\operatorname{dim} \operatorname{Hom}_{\mathrm{D}_{\mathrm{Sp}(2 n)} / \mathrm{Pf}(n)}(r, s) \leq \operatorname{dim}_{\operatorname{Hom}_{\mathrm{Sp}(2 n)}}(r, s) .
$$

This is achieved by combining Lemma 2.14 Lemma 2.16, Theorem 2.18 and Lemma 2.19 below.

We first restrict our attention to the invariant tensors: 
Lemma 2.14. We have isomorphisms of vector spaces

$$
\begin{aligned}
& \operatorname{Hom}_{\mathrm{D}_{\mathrm{Sp}(2 n)} / \mathrm{Pf}(n)}(r, s) \cong \operatorname{Hom}_{\mathrm{D}_{\mathrm{Sp}(2 n)} / \mathrm{Pf}(n)}(0, r+s) \\
& \operatorname{Hom}_{\mathrm{T}_{\mathrm{Sp}(2 n)}}(r, s) \cong \operatorname{Hom}_{\mathrm{T}_{\mathrm{Sp}(2 n)}}(0, r+s) .
\end{aligned}
$$

Let us recall an indexing set for the basis of $\operatorname{Hom}_{\mathrm{T}_{\mathrm{Sp}(2 n)}}(0, r)$ :

Definition 2.15. An $n$-symplectic oscillating tableau of length $r$ (and final shape $\emptyset$ ) is a sequence of partitions

$$
\left(\emptyset=\mu^{0}, \mu^{1}, \ldots, \mu^{r}=\emptyset\right)
$$

such that the Ferrers diagrams of two consecutive partitions differ by exactly one cell and every partition $\mu^{i}$ has at most $n$ non-zero parts.

Lemma 2.16. Hom $_{\mathrm{T}_{\mathrm{Sp}(2 n)}}\left(0, \otimes^{r} V\right)$ has a basis indexed by $n$-symplectic oscillating tableaux.

Proof: This follows immediately from the branching rule for tensoring the defining representation with an irreducible representation of $\operatorname{Sp}(2 n)$, see [8, Theorem II].

Next we exhibit a set of diagrams that span $\operatorname{Hom}_{\mathrm{D}_{\mathrm{Sp}(2 n)} / \mathrm{Pf}^{(n)}}(0, r)$. In fact, this is the key observation.

Definition 2.17. Let $d$ be a diagram in $D(0, r)$. Then an $n$-crossing in $d$ is a set of $n$ distinct strands such that every pair of strands crosses, i.e., $d$ contains strands $\left(a_{1}, b_{1}\right), \ldots,\left(a_{n}, b_{n}\right)$ with $a_{1}<a_{2}<\cdots<$ $a_{n}<b_{1}<b_{2}<\cdots<b_{n}$. The diagram is $(n+1)$-noncrossing if it contains no $(n+1)$-crossing.

Theorem 2.18. The $(n+1)$-noncrossing diagrams form a basis of $\operatorname{Hom}_{\mathrm{D}_{\mathrm{Sp}(2 n)} / \mathrm{Pf}(n)}(0, r)$.

Proof: We only need to show that the set spans. For each $f$ we write $\operatorname{Pf}(f)$ as a rewrite rule. The term that is singled out is the perfect matching of $\mathcal{S}$ in which every pair of strands crosses. The diagrams which cannot be simplified using these rewrite rules are the $(n+1)$-noncrossing diagrams. The procedure terminates because the number of pairs of strands which cross decreases.

We can now use a bijection due to Sundaram [16, Lemma 8.3] to finish the proof of our main theorem.

Lemma 2.19. For all $n$ and $r$ there is a bijection between the set of $n$-symplectic oscillating tableaux of length $r$ and the set of $(n+1)$-noncrossing diagrams in $D(0, r)$.

\section{Cyclic sieving phenomenon}

We now use the results obtained so far to exhibit instances of the cyclic sieving phenomenon:

Definition 3.1. Let $X$ be a finite set and let $\langle\rho\rangle$ be a cyclic group of order $r$ acting on $X$. Let $P(q)$ be a polynomial with non-negative integer coefficients such that

$$
P\left(\zeta^{d}\right)=\left|\left\{x \in X \mid \rho^{d} x=x\right\}\right|,
$$

where $\zeta$ is a primitive $r$-th root of unity. Then the triple

$$
(X, \rho, P(q))
$$

exhibits the cyclic sieving phenomenon. 
Recall that if $\rho: \mathfrak{S}_{r} \rightarrow \operatorname{End}(U)$ is a representation of $\mathfrak{S}_{r}$ then the Frobenius character $\operatorname{ch}(U)$ is the homogeneous symmetric function of degree $r$ given by

$$
\operatorname{ch}(U)=\frac{1}{r !} \sum_{\pi \in \mathfrak{S}_{r}} \operatorname{tr} \rho(\pi) p_{\lambda(\pi)}
$$

where $p_{\lambda}$ is the power sum symmetric function and $\lambda(\pi)$ is the cycle type of $\pi$.

The fake degree, fd, is a linear map from symmetric functions to polynomials in $q$. On the basis of Schur functions it is given by

$$
\mathbf{f d}\left(s_{\lambda}\right)=\sum_{T} q^{\operatorname{maj}(T)}
$$

where the sum is over standard tableaux of shape $T$ and $\operatorname{maj}(T)$ is the major index of the tableau $T$.

A general technique to obtain a cyclic sieving polynomial $P(q)$ is provided by the following result from [19]:

Theorem 3.2. Let $U$ be a representation of $\mathfrak{S}_{r}$ and let $X \subset U$ be a basis which is permuted by the long cycle $c$. Then $(X, c, P(q))$ exhibits the cyclic sieving phenomenon, where

$$
P(q)=\mathbf{f d} \mathbf{c h}(U) .
$$

The most straightforward application of this theorem is to permutation representations of $\mathfrak{S}_{r}$. Here the representation and the basis are given so it remains to determine the Frobenius character and its fake degree polynomial. In general, the action of the symmetric group on the space of invariant tensors is not a permutation representation, that is, there is no basis of the space of invariant tensors that is permuted by the action of $\mathfrak{S}_{r}$.

As mentioned in the introduction, the Frobenius character of $\operatorname{Hom}_{\mathrm{Sp}(V)}\left(W(\mu), \otimes^{r} V\right)$ was obtained in [16] (using combinatorics) and in [17] (using representation theory). For the special case of the invariant tensors, a geometric proof can be found in Procesi [10, Equation 11.5.1.6].

\section{Lemma 3.3.}

$$
\operatorname{ch~} \operatorname{Hom}_{\mathrm{T}(2 n)}(0,2 r)=\sum_{\begin{array}{c}
\lambda \vdash 2 r \\
\text { columns of even length } \\
\ell(\lambda) \leq 2 n
\end{array}} s_{\lambda^{t}} .
$$

Remark 3.4. The partitions indexing the Schur functions appearing in the Frobenius character are all transposed, since we defined $V$ to be an $o d d$ vector space.

Theorem 3.5. Let $X=X(r, n)$ be the set of $(n+1)$-noncrossing perfect matchings on $\{1, \ldots, 2 r\}$ and let $\rho$ be the rotation map acting on $X$. Let

$$
P(q)=\sum_{\substack{\lambda \vdash-2 r \\ \text { columnsof even length } \\ \ell(\lambda) \leq 2 n}} \mathbf{f d} s_{\lambda^{t}}
$$

Then the triple $(X, \rho, P(q))$ exhibits the cyclic sieving phenomenon. 

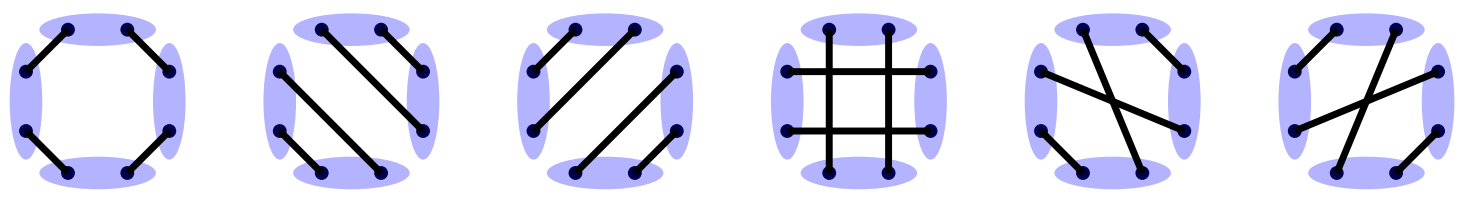

Fig. 1: The six elements of $X(4,2,2)$.

The two extreme cases of Theorem $3.5 n=1$ and $n \geq r$ are known. Putting $n=1, X(r, 1)$ is the set of non-crossing perfect matchings. The corresponding cyclic sieving phenomenon can be found in [12] and [9]. For $n \geq r, X(r, n)$ is the set of all perfect matchings. The Frobenius character of this permutation representation was expanded by Littlewood into Schur functions. Using $\circ$ for plethystic composition, we have

$$
h_{r} \circ h_{2}=\sum_{\substack{\lambda \vdash 2 r \\ \text { rows of even length }}} s_{\lambda} .
$$

Remark 3.6. Let $T$ be an oscillating tableau. The descent set of $T$ is defined in [13] and is denoted by $\operatorname{Des}(T)$. The major index of $T$ is

$$
\operatorname{maj}(T)=\sum_{i \in \operatorname{Des}(T)} i
$$

Then the main result of [13] is that

$$
P(q)=\sum_{T} q^{\mathbf{m a j}(T)}
$$

where the sum is over $n$-symplectic oscillating tableaux of length $2 r$ and weight 0 .

Theorem 3.5 can be generalised. Informally, we consider the set of $k$-regular graphs (with loops prohibited but multiple edges allowed) on $r$ vertices which are also $(n+1)$-noncrossing. More precisely, consider $\{1, \ldots k r\}$ as a cyclically ordered set, partitioned into $r$ blocks of $k$ consecutive elements each. Let $X=X(r, n, k)$ be the set of $(n+1)$-noncrossing perfect matchings of $\{1, \ldots k r\}$ such that there is no pair contained in a block and if two pairs cross then the four elements are in four distinct blocks, see Figure 1 for an example. Finally, let $\rho$ be rotation by $k$ points, i.e., $\rho(i)=i+k-1(\bmod k r)+1$.

For $n>k r$, the set $X(r, n, k)$ is independent of $n$ and can be identified with the set of $k$-regular graphs with multiple edges allowed but loops prohibited. These $k$-regular graphs would normally be considered as a permutation representation of $\mathfrak{S}_{r}$ acting by permuting the vertices. Here the action of $\mathfrak{S}_{r}$ is a linear action on the space with basis $X(r, n, k)$. Example 3.11 below shows that these actions are different.

Lemma 3.7. The invariant tensors corresponding to the diagrams $X(r, n, k)$ form a basis of the space of $\operatorname{Sp}(2 n)$-invariants in the $r$-th tensor power of the $k$-th symmetric power of the defining representation of $\operatorname{Sp}(2 n)$.

The Frobenius character of this representation is given by an application of [20, Theorem 1].

Lemma 3.8. The Frobenius character of the space of $\mathrm{Sp}(2 n)$-invariants in the $r$-th tensor power of the $k$-th symmetric power of the defining representation of $\mathrm{Sp}(2 n)$ is

$$
\sum_{\substack{\lambda \vdash k r \\ \text { colums of even length } \\ \ell(\lambda) \leq 2 n}}\left\langle h_{r}\left(X \cdot e_{k}(Y)\right), s_{\lambda^{t}}(Y)\right\rangle_{Y}
$$


where $e_{k}$ is the $k$-th elementary symmetric function and $\langle,\rangle_{Y}$ denotes the scalar product of symmetric functions with respect to the alphabet $Y$.

Theorem 3.9. Let $X=X(r, n, k)$ and let $\rho$ be the rotation map. Define $P(q)$ to be the fake degree of the symmetric function (3). Then $(X, \rho, P)$ exhibits the cyclic sieving phenomenon.

The case $k=1$ is Theorem 3.5 and the case $k=2$ is related to the invariant tensors of the adjoint representation in [5]. The case $n=1$ is implicit in [2] and the case $n=2$ is related to the $C_{2}$ webs in [6].

Instead of the $k$-th symmetric power of $V$ we can also consider the $k$-th fundamental representation of $\operatorname{Sp}(2 n)$. Again using [20, Theorem 1] we obtain the following expression for the Frobenius character of its tensor powers.

Lemma 3.10. The Frobenius character of the space of $\operatorname{Sp}(2 n)$-invariants in the $r$-th tensor power of the $k$-th fundamental representation of $\operatorname{Sp}(2 n)$ is

$$
\sum_{\substack{|\lambda| \leq k r \\ \text { columns of even length } \\ \ell(\lambda) \leq 2 n}}\left\langle h_{r}\left(X \cdot\left(h_{k}-h_{k-2}\right)(Y)\right), s_{\lambda^{t}}(Y)\right\rangle_{Y}
$$

Note that in general, this space cannot have a basis invariant under cyclic rotation. For example, for $n=1, k=3$ and $r=2$ its Frobenius character evaluates to $s_{1,1}$ with fake degree polynomial equal to $q$, which is not a cyclic sieving polynomial.

However, let us compare the two Frobenius characters for $n>k r$. Setting $H=1+h_{1}+h_{2}+\cdots$ we obtain for the symmetric powers

$$
\left\langle h_{r}\left(X \cdot e_{k}(Y)\right), H\left(h_{2}(Y)\right)\right\rangle_{Y}
$$

whereas for the fundamental representation the expression becomes

$$
\left\langle h_{r}\left(X \cdot\left(h_{k}-k_{k-2}\right)(Y)\right), H\left(h_{2}(Y)\right)\right\rangle_{Y}
$$

For $n>k r$, the set $X(r, n, k)$ is independent of $n$ and can be identified with the set of $k$-regular graphs with multiple edges allowed but loops prohibited. This is a species whose Frobenius character is given by equation (6), see Travis [18].

The action of the symmetric group in the first case is defined using a sign and is therefore not a permutation representation, as illustrated by the following example.

Example 3.11. Putting $k=2$ and $r=6$, the formula (5) for invariant tensors gives

$$
\frac{1}{72}\left(13 p_{1^{6}}+12 p_{21^{4}}+63 p_{2^{2} 1^{2}}+54 p_{2^{3}}+4 p_{31^{3}}-12 p_{321}+28 p_{3^{2}}+18 p_{41^{2}}+36 p_{42}+36 p_{6}\right)
$$

and the formula (6) for regular graphs gives

$$
\frac{1}{72}\left(13 p_{1^{6}}+24 p_{21^{4}}+63 p_{2^{2} 1^{2}}+54 p_{2^{3}}+4 p_{31^{3}}+12 p_{321}+28 p_{3^{2}}+18 p_{41^{2}}+36 p_{42}+36 p_{6}\right)
$$




\section{Partitions and directed matchings}

The discussion in the previous sections has been about the Brauer category and the categories of invariant tensors for the vector representation of $\operatorname{Sp}(2 n)$. In this section we discuss two other examples where we have a diagram category which is related to a sequence of categories of invariant tensors.

In the first example we consider the linear representation $V$ of the symmetric group $\mathfrak{S}_{n}$ associated to its defining representation. The diagram category in this case is the partition category studied in [4].

Let $I(r, n)=\operatorname{Hom}_{\mathfrak{S}_{n}}\left(\mathbb{C}, \otimes^{r} V\right)$ be the space of invariant tensors with the action of $\mathfrak{S}_{r}$ and let $X(r, n)$ be the set of set partitions of $\{1, \ldots, r\}$ into at most $n$ blocks. Then there is a map $X(r, n) \rightarrow I(r, n)$ such that the image is a basis. Furthermore this basis is preserved by the action of $\mathfrak{S}_{r}$. It follows that the Frobenius character is the homogeneous component of degree $r$ in $h_{n} \circ H$, where $H=1+h_{1}+h_{2}+\cdots$.

As in the case of the Brauer category, this result can be generalised, by considering the tensor powers of the $k$-th symmetric power of the defining representation. The resulting set $X(r, n, k)$ is then the set of multiset partitions into at most $n$ blocks of the multiset $\{1, \ldots, 1,2, \ldots, 2, \ldots, r, \ldots, r\}$, each label occuring precisely $k$ times. The symmetric group $\mathfrak{S}_{r}$ acts on this set by permuting the labels. The Frobenius character can be obtained using [20, Theorem 1] or alternatively with the calculus of species and is given by

$$
\left\langle h_{r}\left(X \cdot h_{k}(Y)\right), h_{n}(H(Y))\right\rangle_{Y} .
$$

In the second example we consider the adjoint representation $V$ of the general linear group GL( $n)$. In this case the diagrams are Brauer diagrams in which every edge is directed.

For $n \geq 2 r$ let $X(r)$ be the set of permutations with the conjugation action of $\mathfrak{S}_{r}$. Its Frobenius character is obtained in [15] using character calculations. Our methods give a simple proof. The Frobenius character is

$$
\sum_{\lambda \vdash r} p_{\lambda}=\sum_{\lambda \vdash r} s_{\lambda} * s_{\lambda}
$$

where $*$ denotes the Kronecker or inner product.

Let $I(r, n)=\operatorname{Hom}_{\mathrm{GL}(n)}\left(\mathbb{C}, \otimes^{r} V\right)$ be the space of invariant tensors with the action of $\mathfrak{S}_{r}$. Considering the diagrams we obtain the new result that the Frobenius character of this representation is

$$
\sum_{\substack{\lambda \vdash r \\ \ell(\lambda) \leq n}} s_{\lambda} * s_{\lambda}
$$

However, for $n<2 r$ it is an open problem to determine a set $X(r, n)$ with a map $X(r, n) \rightarrow I(r, n)$ such that the image is a basis and is preserved by the action of the long cycle. So we are not able to exhibit an example of the cyclic sieving phenomenon in this case.

\section{References}

[1] R. Brauer. "On algebras which are connected with the semisimple continuous groups". In: Ann. of Math. (2) 38.4 (1937), pp. 857-872.

[2] I. B. Frenkel and M. G. Khovanov. "Canonical bases in tensor products and graphical calculus for $U_{q}\left(\mathfrak{s l}_{2}\right)$ ”. In: Duke Math. J. 87.3 (1997), pp. 409-480.

[3] F. Gavarini. "A Brauer algebra-theoretic proof of Littlewood's restriction rules”. In: J. Algebra 212.1 (1999), pp. 240-271. 
[4] T. Halverson and A. Ram. "Partition algebras". In: European J. Combin. 26.6 (2005), pp. 869-921.

[5] P. Hanlon. "On the decomposition of the tensor algebra of the classical Lie algebras". In: $A d v$. in Math. 56.3 (1985), pp. 238-282.

[6] G. Kuperberg. "Spiders for rank 2 Lie algebras". In: Comm. Math. Phys. 180.1 (1996), pp. 109151.

[7] G. I. Lehrer and R. B. Zhang. The Brauer Category and Invariant Theory. 2012. arXiv: arXiv: 1207.5889 [math.RT]

[8] D. E. Littlewood. "Products and plethysms of characters with orthogonal, symplectic and symmetric groups". In: Canad. J. Math. 10 (1958), pp. 17-32.

[9] T. K. Petersen, P. Pylyavskyy, and B. Rhoades. "Promotion and cyclic sieving via webs". In: $J$. Algebraic Combin. 30.1 (2009), pp. 19-41.

[10] C. Procesi. Lie groups. Universitext. An approach through invariants and representations. Springer, New York, 2007, pp. xxiv+596. ISBN: 978-0-387-26040-2; 0-387-26040-4.

[11] V. Reiner, D. Stanton, and D. White. "The cyclic sieving phenomenon”. In: J. Combin. Theory Ser. A 108.1 (2004), pp. 17-50.

[12] B. Rhoades. "Cyclic sieving, promotion, and representation theory". In: J. Combin. Theory Ser. A 117.1 (2010), pp. 38-76.

[13] M. Rubey, B. E. Sagan, and B. W. Westbury. "Descent sets for symplectic groups". In: J. Algebraic Combin. 40.1 (2014), pp. 187-208.

[14] M. Rubey and B. Westbury. A combinatorial approach to classical representation theory. 2014. arXiv: 1408.3592 [math.RT]

[15] R. P. Stanley. "The stable behavior of some characters of $\operatorname{SL}(n, \mathbf{C})$ ". In: Linear and Multilinear Algebra 16.1-4 (1984), pp. 3-27.

[16] S. Sundaram. On the combinatorics of representations of the symplectic group. Thesis (Ph.D.)Massachusetts Institute of Technology. ProQuest LLC, Ann Arbor, MI, 1986, (no paging).

[17] T. Tokuyama. "Highest weight vectors associated with some branchings". In: The Arcata Conference on Representations of Finite Groups (Arcata, Calif., 1986). Vol. 47. Proc. Sympos. Pure Math. Providence, RI: Amer. Math. Soc., 1987, pp. 541-545.

[18] L. E. Travis. Graphical enumeration: A species-theoretic approach. Thesis (Ph.D.)-Brandeis University. ProQuest LLC, Ann Arbor, MI, 1999, p. 57. ISBN: 978-0599-16906-7.

[19] B. W. Westbury. Invariant tensors and the cyclic sieving phenomenon. 2010. arXiv: arXiv: 0912.1512 [math.RT]

[20] B. W. Westbury. On enumeration in classical invariant theory. 2014. arXiv: arXiv:1402.6111 [math.RT].

[21] Hermann Weyl. The classical groups. Princeton Landmarks in Mathematics. Their invariants and representations, Fifteenth printing, Princeton Paperbacks. Princeton, NJ: Princeton University Press, 1997, pp. xiv+320. ISBN: 0-691-05756-7. 